\title{
ANÁLISE DE FLUXO DE NUTRIENTES COM FOCO NO SETOR DE SANEAMENTO DO ESTADO DA BAHIA
}

\author{
$\underline{\text { Ana Caroline Bastos }} \mathbf{1}$; Eduardo Cohim²; Samuel Sipert ${ }^{3}$ \\ 1. Bolsista PROBIC/UEFS, Graduanda em Engenharia Civil, Universidade Estadual de Feira de Santana, e-mail: \\ anacarolinebxstos@gmail.com \\ 2. Orientador, Departamento de Tecnologia, Universidade Estadual de Feira de Santana, e-mail: edcohim@gmail.com \\ 3. Aluno do Programa de Pós-Graduação em Engenharia Civil e Ambiental, Universidade Estadual de Feira de Santana, e- \\ mail: $\underline{\text { ssipert@ hotmail.com }}$
}

PALAVRAS-CHAVE: segurança alimentar; análise de nutrientes; saneamento.

\section{1- INTRODUÇÃO}

De acordo com a United Nations (2015), espera-se que a população mundial alcance 8,5 bilhões de pessoas até 2030, aumentando para 9,7 bilhões em 2050. Esse crescimento populacional aumentará a demanda de alimentos e poderá induzir conflitos de demanda e oferta, colocando em discussão a segurança alimentar. Levando em consideração a interdependência entre alimento, água e energia (nexus), a elaboração de estratégias que promovam a sustentabilidade da sociedade se torna imprescindível.

$\mathrm{O}$ uso de fertilizantes químicos nos sistemas agrícolas foi fundamental para suprir a crescente demanda mundial por alimentos. Entretanto, a grande produção de nitrogênio e fósforo reativos através de processos que possuem uma alta demanda de energia e de recursos naturais, também foi a principal causa das mudanças dos ciclos biogeoquímicos terrestres, os quais já ultrapassaram os limites planetários propostos por Rockstrom et al. (2009) para uma operação sustentável.

Além disso, nosso modelo convencional de saneamento desperdiça uma grande quantidade de nutrientes, os quais são despejados nos corpos hídricos e passam a atuar como poluentes (BOUWMAN et al., 2013). Esse tipo de gestão demanda altos investimentos financeiros, ao mesmo tempo em que contribui para a superexploração de fontes renováveis de água e o empobrecimento do solo.

Este estudo faz uma análise de fluxo de materiais (AFM) visando obter um melhor entendimento à respeito do gerenciamento de recursos. Através do monitoramento e quantificação de cada fluxo, conseguimos ter uma noção do quanto é desperdiçado e prever os benefícios de ações que, a partir de uma visão que considere os resíduos como recursos, garantam o fechamento do ciclo de nutrientes.

\section{2- METODOLOGIA}

O balanço de entradas e saídas de nutrientes se limitou apenas estado da Bahia, com valores referentes ao ano-base de 2008, devido à disponibilidade de informações. A população para o ano de estudo foi calculada em 13820370 habitantes, a partir dos dados do Censo do ano 2000, utilizando uma taxa média geométrica de crescimento anual de $0,70 \%$.

\section{1- Dados de entrada}

Os fluxos de entrada de nitrogênio $(\mathrm{N})$, fósforo $(\mathrm{P})$ e potássio $(\mathrm{K})$ através dos alimentos foram calculados a partir das quantidades de alimentos adquiridos por pessoa, disponível em uma tabela da Food and Agriculture Organization of the United Nations (FAO), e dos teores de nutrientes descritos em diversas publicações, dentre as quais, destacase a Tabela Brasileira de Composição de Alimentos - TACO (NEPA, 2011).

\section{2- Dados de saída}

Para as correntes de excreção, foram considerados os valores propostos por Friedler et al. (2013), sendo 11, 0,93 e 2,6 (g/hab.dia) e 1,5, 0,6 e 0,9 (g/hab.dia) a geração per capita de 
N, P e K para urina e fezes, respectivamente. É importante destacar a grande variabilidade desses dados, tendo em vista que são influenciados pelos hábitos de alimentação e saúde dos indivíduos, bem como fatores sociais, econômicos e ambientais.

A taxa de geração per capita de Resíduos Sólidos Orgânicos (RSO), por sua vez, foi estimada em 0,621 kg/hab.dia, com base na quantidade per capita de Resíduos Sólidos Urbanos (RSU) referente à macrorregião do Nordeste de 1,21 kg/hab.dia (ABRELPE, 2008), e na composição gravimétrica do RSU coletado no Brasil, com fração orgânica de 51,4\%, de acordo com o Plano Nacional de Resíduos Sólidos (PNRS) (BRASIL, 2011). Os teores de nutrientes para o RSO foram extraídos de Wendland (2009), com valores de 5,3, 0,53 e 1,8 $\mathrm{g} / \mathrm{kg}$ de RSO, para N, P e K, respectivamente.

\section{3- Reconciliação de dados}

O modelo de análise de fluxo de materiais segue o princípio fundamental de conservação de massa, porém, a existência de fluxos conflitantes neste estudo tornou necessário o uso da técnica de reconciliação de dados para fechamento do balanço.

Devido à falta de medição e de informação à respeito das incertezas dos dados, adotou-se a técnica proposta por Martins et al. (2010), que utiliza o conceito de qualidade de informação (QI) para representar o nível de incerteza relacionado aos fluxos. Por fim, os fluxos reconciliados foram transferidos para o software STAN (subSTance flow ANalysis), com a finalidade de expor melhor os resultados do estudo.

\section{3- RESULTADOS E DISCUSSÃO}

A Tabela 1 apresenta as quantidades de alimento e de cada nutriente consumidos por pessoa no Brasil, em 2008. Considerando a população calculada para o estado da Bahia no ano em estudo, o fluxo de entrada de nitrogênio no volume de controle é de $9,37 \times 10^{7} \mathrm{~kg}$, o de fósforo é de $1,04 \times 10^{7} \mathrm{~kg}$ e o de potássio corresponde a $2,32 \times 10^{7} \mathrm{~kg}$. A Tabela 2 resume os valores das correntes de saída para essa população e o resultado do balanço, após a reconciliação de dados, se encontra nas figuras $1 \mathrm{a}, 1 \mathrm{~b}$ e 2 .

Tabela 1. Aquisição alimentar per capita em 2008.

\begin{tabular}{llllll}
\hline $\begin{array}{l}\text { Grupo } \\
\text { alimentar }\end{array}$ & $\begin{array}{l}\text { Qtde. de } \\
(\mathbf{k g})\end{array}$ & alimento & $\begin{array}{l}\text { Qtde. de } \\
(\mathbf{k g})\end{array}$ & $\begin{array}{l}\text { Qtde. de } \\
(\mathbf{k g})\end{array}$ & $\begin{array}{l}\text { Qtde. de K / } \\
(\mathbf{k g})\end{array}$ \\
\hline Cereais & $1,14 \mathrm{E}+02$ & $1,83 \mathrm{E}+00$ & $3,04 \mathrm{E}-01$ & $3,96 \mathrm{E}-01$ \\
Raízes & $6,58 \mathrm{E}+01$ & $1,30 \mathrm{E}-01$ & $1,90 \mathrm{E}-02$ & $1,44 \mathrm{E}-01$ \\
Açúcares & $1,51 \mathrm{E}+01$ & $4,75 \mathrm{E}-02$ & $7,17 \mathrm{E}-03$ & $8,68 \mathrm{E}-02$ \\
Leguminosas & $1,67 \mathrm{E}+01$ & $5,42 \mathrm{E}-01$ & $6,47 \mathrm{E}-02$ & $2,03 \mathrm{E}-01$ \\
Nozes & $4,69 \mathrm{E}-01$ & $1,08 \mathrm{E}-02$ & $1,86 \mathrm{E}-03$ & $2,50 \mathrm{E}-03$ \\
Oleaginosas & $1,94 \mathrm{E}+01$ & $3,28 \mathrm{E}-01$ & $4,01 \mathrm{E}-02$ & $1,21 \mathrm{E}-01$ \\
Vegetais & $4,73 \mathrm{E}+01$ & $5,09 \mathrm{E}-02$ & $6,07 \mathrm{E}-03$ & $5,16 \mathrm{E}-02$ \\
Frutas & $1,15 \mathrm{E}+02$ & $1,59 \mathrm{E}-01$ & $1,75 \mathrm{E}-02$ & $1,89 \mathrm{E}-01$ \\
Estimulantes & $6,94 \mathrm{E}+00$ & $1,19 \mathrm{E}-01$ & $8,94 \mathrm{E}-03$ & $8,35 \mathrm{E}-02$ \\
Especiarias & $2,09 \mathrm{E}-01$ & $3,36 \mathrm{E}-04$ & $4,07 \mathrm{E}-05$ & $5,32 \mathrm{E}-04$ \\
Carnes & $8,62 \mathrm{E}+01$ & $2,68 \mathrm{E}+00$ & $1,49 \mathrm{E}-01$ & $2,14 \mathrm{E}-01$ \\
Ovos & $7,61 \mathrm{E}+00$ & $1,63 \mathrm{E}-01$ & $1,25 \mathrm{E}-02$ & $1,14 \mathrm{E}-02$ \\
Leite & $1,29 \mathrm{E}+02$ & $7,24 \mathrm{E}-01$ & $1,20 \mathrm{E}-01$ & $1,73 \mathrm{E}-01$ \\
\hline Total & $6,24 \mathrm{E}+02$ & $6,78 \mathrm{E}+00$ & $7,51 \mathrm{E}-01$ & $1,68 \mathrm{E}+00$ \\
\hline
\end{tabular}

Tabela 2. Saída de nutrientes em 2008.

\begin{tabular}{llll}
\hline Corrente & N / $(\mathrm{kg})$ & $\mathbf{P} /(\mathrm{kg})$ & $\mathrm{K} /(\mathrm{kg})$ \\
\hline
\end{tabular}




\begin{tabular}{llll}
\hline Fezes & $7,57 \mathrm{E}+06$ & $3,03 \mathrm{E}+06$ & $4,54 \mathrm{E}+06$ \\
Urina & $5,55 \mathrm{E}+07$ & $4,54 \mathrm{E}+06$ & $1,31 \mathrm{E}+07$ \\
RSO & $1,66 \mathrm{E}+07$ & $1,57 \mathrm{E}+06$ & $5,64 \mathrm{E}+06$ \\
\hline Total & $7,97 \mathrm{E}+07$ & $9,13 \mathrm{E}+06$ & $2,33 \mathrm{E}+07$ \\
\hline
\end{tabular}
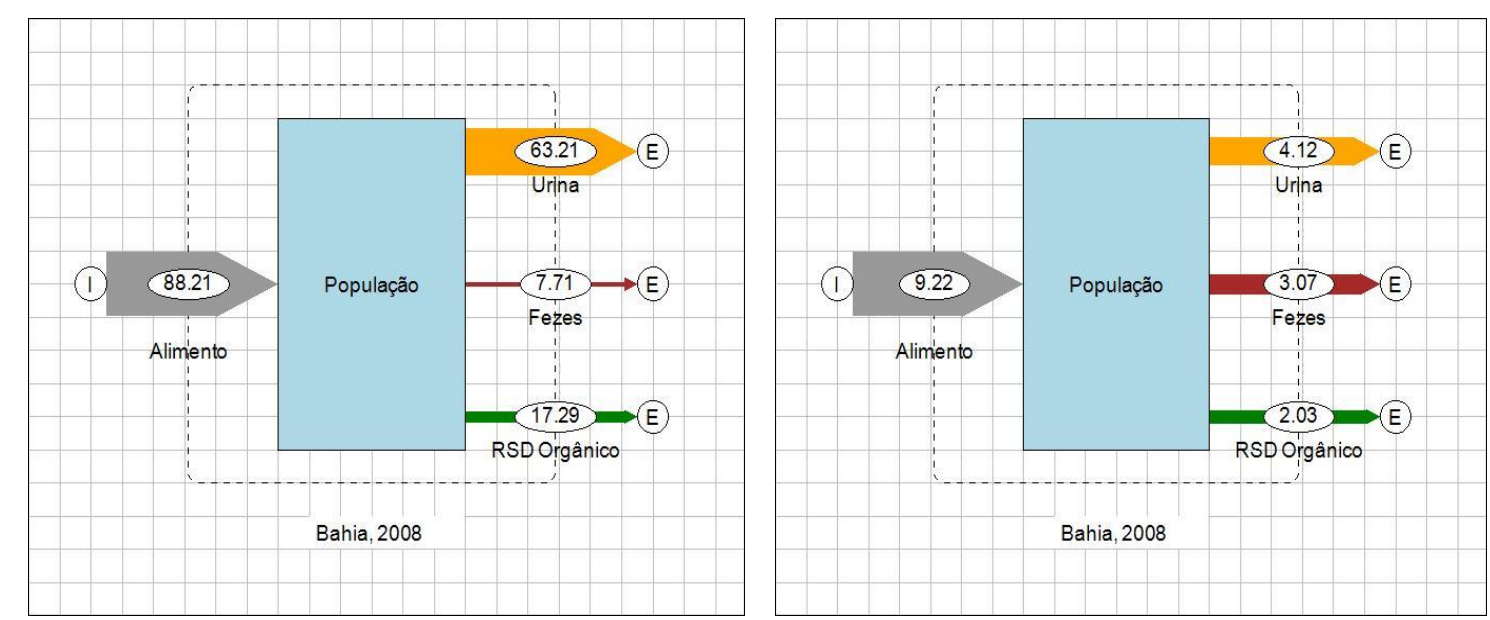

Figura 1a: Fluxos de Nitrogênio, em milhares de toneladas, para a população baiana.

Figura 1b: Fluxos de Fósforo, em milhares de toneladas, para a população baiana.

Analisando a Figura 1a, fica evidente a importância da recuperação da urina humana no que diz respeito ao aproveitamento de nutrientes. Apesar de representar apenas $1 \%$ do volume total direcionado às estações de tratamento de esgoto (Larsen et al., 2001), essa corrente contribuiu com cerca de $89 \%$ do nitrogênio contido no efluente. É importante destacar a grande variabilidade desses dados, tendo em vista que são influenciados pelos hábitos de alimentação e saúde dos indivíduos, bem como fatores sociais, econômicos e ambientais.

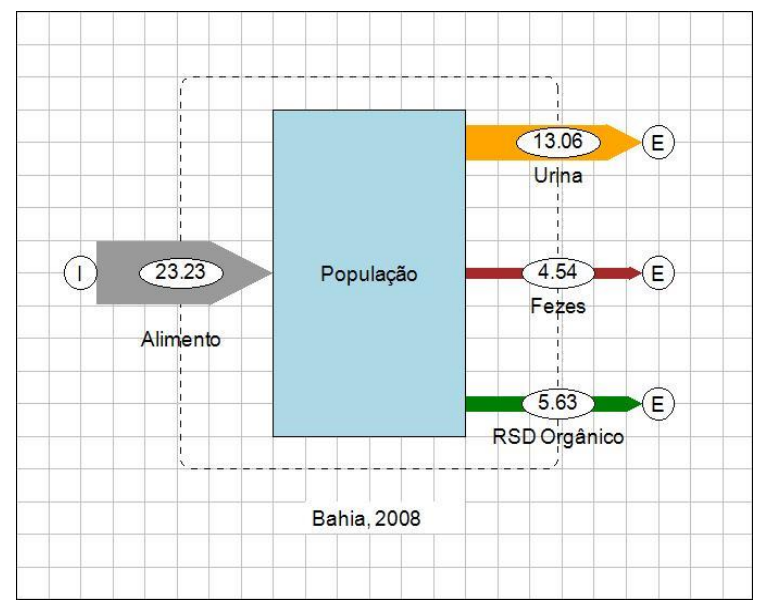

Figura 2: Fluxos de Potássio, em milhares de toneladas, para a população baiana.

Tomando como base os dados de consumo de fertilizantes determinados por Manos et al. (2013) e Souza et al. (2009), a quantidade de nutrientes saindo do sistema seria mais que suficiente para a fertilização de mais de 300000 hectares de milho ou mandioca por ano. Isso representaria um grande impacto social, tendo em vista que pequenos produtores rurais geralmente não tem acesso a fertilizantes químicos, podendo fazer uso dessa fonte alternativa.

Além disso, a recuperação desses nutrientes também geraria uma economia de energia pela diminuição da produção de fertilizantes químicos. Com base em indicadores de demanda energética citados por Harvey (2010), essa economia poderia chegar a 2,03 TWh, quase o 
triplo do valor de energia elétrica consumido pelos sistemas de abastecimento de água e esgotamento sanitário para o Estado da Bahia em 2008 (0,64 TWh) (BRASIL, 2010).

\section{4- CONCLUSÃO}

Podemos notar através dos resultados desse estudo a importância do setor de saneamento no que diz respeito ao acúmulo de nitrogênio, fósforo e potássio. $\mathrm{O}$ redirecionamento desses fluxos para um aproveitamento agrícola evitaria a contaminação de ecossistemas, reduziria o gasto energético com a retirada de nutrientes do esgoto de estações de tratamento, além de minimizar os custos econômicos com a importação de fertilizantes e amenizar o déficit de nutrientes do solo. Apesar da baixa qualidade dos dados, os números mostram pela ordem de grandeza a importância desse tipo de estudo no que diz respeito à um melhor gerenciamento dos recursos.

\section{5- REFERENCIAS}

ASSOCIAÇÃO DAS EMPRESAS DE LIMPEZA PÚBLICA (ABRELPE). 2008. Panorama de Resíduos Sólidos no Brasil.

BOUWMAN, L. et al. 2013. Exploring global changes in nitrogen and phosphorus cycles in agriculture induced by livestock production over the 1900-2050 period. Proc Natl Acad Sci U $S A$, v. 110 , n. 52, p. 20882-20887.

BRASIL. 2011 [online]. Plano Nacional de Resíduos Sólidos. Disponível em: <www.mma.gov.br/estruturas/253/_publicacao/253_publicacao02022012041757.pdf>.

BRASIL. 2010. Sistema Nacional de Informações sobre Saneamento: diagnóstico dos serviços de água e esgotos - 2008. Brasília: MCIDADES/SNSA.

FRIEDLER, et al. Wastewater Composition. Editors: LARSEN, T. A. et al. 2013. Source Separation and Descentralization of Wastewater Management. IWA Publishing.

HARVEY, L.D.D. 2010. Energy efficiency and the demand for energy services: Energy and the new reality. 1 ed. Earthscan-London.

LARSEN, T. A. et al. 2001. Re-engineering the toilet for sustainable wastewater management. Environmental Science Technologies.

MANOS M. G. L., OLIVEIRA M. G. C. \& MARTINS C. R. 2013. Informações Técnicas para o Cultivo do Feijoeiro Comum na Região Nordeste Brasileira 2013-2014. 17ª Reunião de Comissão Técnica Norte/Nordeste Brasileira de Feijão - CNTNBF, Embrapa Tabuleiros Costeiros.

MARTINS, M. A. F. et al. 2010. New objective function for data reconciliation in water balance from industrial processes. J. Cleaner Prod.

NÚCLEO DE ESTUDOS E PESQUISAS EM ALIMENTAÇÃO (NEPA). 2006. TACO Tabela Brasileira de Composição de Alimentos. 4.ed. Campinas: Nepa-Unicamp.

ROCKSTRÖM, Johan et al. 2009. A safe operating space for humanity. Nature, v. 461, n. 7263, p. 472.

SOUZA L. S., SILVA J. \& SOUZA L. D. 2009. Recomendação de Calagem e Adubação para o Cultivo da Mandioca. Comunicado Técnico 133, Embrapa Mandioca e Fruticultura Tropical, Cruz das Almas, Brasil.

UNITED NATIONS (UN). 2015 [online]. World Population Prospects: The 2015 Revision, Key Findings and Advance Tables. Department of Economic and Social Affairs, Population Division.

<http://esa.un.org/unpd/wpp/Publications/Files/Key_Findings_WPP_2015.pdf $>$. Homepage:

WENDLAND, C. 2009. Anaerobic digestion of blackwater and kitchen refuse. Technische Universität Hamburg. 